\title{
Carpal Tunnel Syndrome: Causes, Prevention, Rehabilitation and Computer-Aided, Game-Based Physiotherapy
}

\author{
${ }^{1}$ Diana Tsilidi, ${ }^{2}$ loannis Pachoulakis, ${ }^{3}$ Anastasia Analyti \\ ${ }^{1,2}$ Department of Informatics Engineering, Technological Educational Institute of Crete \\ Heraklion, Crete, Greece; \\ ${ }^{3}$ Institute of Computer Science, Foundation for Research and Technology - Hellas (FORTH) \\ Vassilika Vouton, Heraklion, Crete, Greece; \\ dtsilidi@gmail.com; ip@ie.teicrete.gr; analyti@ics.forth.gr
}

\begin{abstract}
Carpal Tunnel Syndrome (CTS) is caused by improper computer posture and usage of the arms and hands. It expresses a conglomerate of symptoms caused by the compression of the median nerve as it passes through the carpal tunnel of the wrist. These include pain, numbness and tingling in affected parts of the hand. CTS treatment may take the form of surgery and/or physiotherapy. This paper revisits the causes of CTS and the common prevention tactics and focuses on the emerging role of rehabilitation via computer-aided, game-based physiotherapy which can augment a more traditional exercise curriculum.
\end{abstract}

Keywords: Carpal tunnel syndrome (CTS); Computer-aided rehabilitation; Game-based Rehabilitation.

\section{Introduction}

The Carpal Tunnel Syndrome (CTS) is a medical condition in which the median nerve is compressed as it travels through the wrist's carpal tunnel causing pain, numbness and tingling in parts of the hand that receive sensation from the median nerve [1]. It has also been associated with conditions such as obesity, hypothyroidism, arthritis, diabetes and trauma. The syndrome was at first described by Sir James Paget, after a case of medial nerve compression at the wrist following a fracture of the distalradius. The principles of CTS were established in 1950, when George Phalen reported for the very first time this relatively unknown condition at the 99th Annual Meeting of the American Medical Association. To comprehend CTS, one must first get familiarized with the relevant wrist anatomy and the tunnel that is shaped by the carpal bones as shown in Error! Reference source not found.. The wrist has an incurved contour that consists of eight bones, forming the joint, on the surface of the flexor, the muscle which bends the limb by its contraction, and is covered by the flexor retinaculum or transverse carpal ligament, a fibrous band on the palmar side of the hand [2].

In effect, the carpal tunnel is confined by the bony carpus and the transverse carpal ligament (flexor retinaculum). The latter connected to the tubercle of the scaphoid carpal bone, the ridge of the trapezium carpal bone and the ulnar side of the hook (small projection of the bone) of the hamate and pisiform carpal bones. The flexor muscles of the fingers pass through the carpal tunnel, while the median nerve is located under the transverse carpal ligament [2].

In conclusion, the wrist consists of small bones, the carpal bones, which form the carpal tunnel. The median nerve, which controls the sensations in the palm and fingers, as well as the tendons pass through this tunnel from the forearm into the hand. The pressure on the median nerve can be 
sometimes caused by swelling and irritation of the tendons, thus producing the symptoms of the CTS. The affected hand is usually the dominant hand of the patient, although it also quite possible to observe symptoms of CTS in both hands [2].
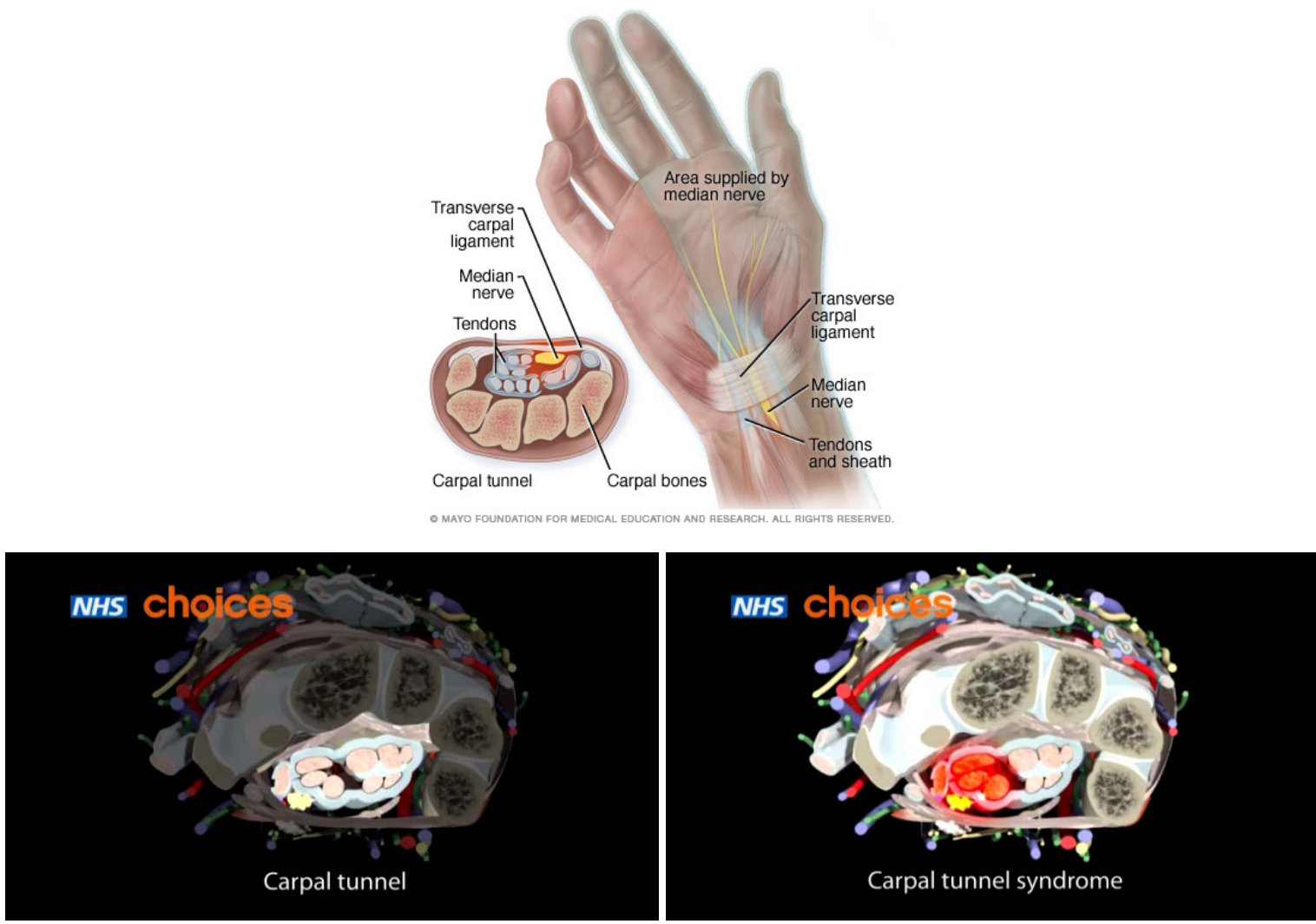

Figure 1: Top: Wrist anatomy (adopted from [3]); Bottom Left: Physiological ligaments in the carpal tunnel; Bottom right: Inflamed ligaments in red in the carpal tunnel (both images adopted from [4])

Treatment of CTS aims at restoring normal hand functionality to allow patients to resume their usual daily activities as well as prevent further/additional nerve damage and muscle loss in the fingers and hands. Mainstream treatment options include wrist splinting and immobilization, administration of medicines such as corticosteroids and anti-inflammatory drugs that relieve pain and reduce inflammation, physical therapy and, finally, surgery as a last resort measure, when other treatments fail.

\section{Origins of the Carpal Tunnel Syndrome}

The compression of the median nerve occurs at the carpal tunnel as the result of an incompatibility between the relative size of the canal and the volume of its content, the space occupied by the transit tendons and the median nerve. Studies, including the measurement of the inter-carpal canal pressure of CTS patients, have shown significantly increased mean pressure in the carpal canal. The most significant mean carpal canal pressure increment was observed during the wrist extension and slightly less, when wrist flexion was performed, while an immediate pressure decrease was observed during carpal tunnel release. Therefore, the syndrome is usually caused by several different conditions. The space inside the carpal canal can be reduced by processes including tenosynovitis of the flexor tendons, fracture or dislocation of the carpus and carpometacarpal joints and Colles' fracture. These conditions may also cause post-traumatic scarring or fibrosis inside the carpal tunnel. Rheumatoid arthritis, amyloid deposition, granoulomatous infection and gout are some of the inflammatory processes that contribute to reduced volume inside the carpal tunnel and produce a proliferative tenosynovitis with 
hyperplastic synovium [2]. Other causes for the decreased space within the carpal canal can be tumors of the median nerve, disorders like acromegaly or hypothyroidism, pregnancy, diabetes mellitus, and lupus erythematosus. Some of these conditions may also increment the extracapsular fluid retention and produce swelling of the soft tissue.

It has been observed that women are three times more likely to have CTS than men. However, the reason for this increased frequency in women population is not known yet. Some of the theories mention that it may be caused by the difference in the size of the wrist bones, which are naturally smaller in most women. This means that the space that is formed by the carpal bones, through which the nerves and tendons pass, is tighter. Other factors that are considered by the scientists are the genetic links that make it more likely for women to have musculoskeletal injuries and the strong hormonal changes.

Certain health problems can also contribute to the onset of CTS, although the exact origins may remain unknown. Factors that raise the chances of developing CTS include genetic predisposition, wrist injury causing swelling and pressure on the nerve or repetitive movements of the hands (computer users, assembly line workers, carpenters, musicians, athletes etc.). Additional women-specific factors contributing to the development of CTS relate to hormonal changes, pregnancy and menopause. In these cases the wrist structure may become enlarged and add pressure on the nerve, but in many cases the symptoms fade after the condition passes. Another rare cause of CTS can be breast cancer, since often after mastectomy the build-up of fluids raises beyond the ability of lymph system to drain it, causing pain and swelling of the arm and pressuring the nerve.

In detail, the most significant and common medical conditions that lead to CTS can be classified into the groups mentioned below:

- Swelling-tenosynovitis of the flexor tendons of the hand due to extensive hand use (often observed to farmers, builders, hairdressers, drivers or wheeled machine operators).

- Swelling of the tendon due to hormonal disorders such as disorders of the thyroid gland and ovarian (menopause or pregnancy).

- All of the above conditions, causing swelling of the sheaths and tendons within the carpal tunnel leading to an increase of pressure within. Initially the pressure affects small vessels of perineural and then the whole nerve that results to conduction disorder. The nerve swells and presents dotted macroscopic bleeding. In chronic cases of this neglected condition of the wrist the medium nerve presents intraneural fibrosis and permanent functional impairment.

- Rarely the syndrome is caused by diabetes or a congenital narrowing of the carpal tunnel.

- In many cases CTS is a result of incorrect positioning of the wrist when typing on a computer. That is why the newer keyboards have wrist support extensions. The disease has also been called journalistic and "typists disease" or "disease of the computer operator."

- In recent years it has been discovered that the intake of the drug Tamoxifen and Arimidex may also lead to CTS.

- In rare cases, CTS coexists with median nerve pressure on the forearm, by the round pronator muscle.

- They have been rare but existing cases of CTS caused by tumors and CTS amyloidosis of histological lesions.

According to [5], the main causes and contributing factors to CTS are:

- Aberrant Anatomy: anomalous flexor tendons, congenitally small carpal canal, ganglionic cysts, lipoma, proximal lumbrical muscle insertion, thrombosed artery. 
- Infections: lyme disease, mycobacterial infection, septic arthritis.

- Inflammatory conditions such as connective tissue disease, gout or pseudo-gout, rheumatoid arthritis and nonspecific flexor tenosynovitis (the most common cause of CTS).

- Metabolic conditions such as diabetes, hypothyroidism / hyperthyroidism, acromegaly, amyloidosis.

- Increased canal volume: congestive heart failure, edema, obesity, pregnancy.

It has also been observed that smokers with CTS have worse symptoms and usually recover more slowly than non-smokers.

\section{Symptoms of Carpal Tunnel Syndrome}

Proper CTS diagnosis entails the observation of the appropriate symptoms in conjunction with abnormal nerve conduction test. The telltale symptoms of CTS are burning pain in the volar aspects of both hands, numbness in the fingers and tingling, which often become worse after work and at night and awakens patients from their sleep. Nighttime symptoms are prominent in $50-70 \%$ of the patient population. Shaking the wrist down may relieve the symptoms. Symptoms are commonly localized to the thumb and the first two or three fingers, often accompanied by burning pain in the palm side of the wrist (although this sensation of pain, numbness and tingling may also expand to the forearm, elbow, and especially the shoulder in the form of aching pain. In severe cases, patients may even lose the hand dexterity because of decreased grip strength and thenar muscle atrophy. Ulnar neuropathy or C-8 radiculopathy should be considered in cases where the nerve symptoms are observed only in the fourth and fifth fingers, whereas proximal symptoms, especially tingling in the radial hand combined with elbow side pain may point towards a possible C-6 radiculopathy.

Signs and symptoms observed during the physical examinations may often be negligible or confusing. Some of the diagnosis methods, like Hoffmann-Tinel's sign, which is described as paresthesias expanding in the median nerve distribution with tapping on the wrist or over the median nerve, and Phalen's sign, which involves paresthesia radiating in a median nerve distribution, but within 60 seconds of sustained flexion of the wrist, are frequently described, though are not specific enough for the diagnosis of CTS. The presence of these signs reinforces the existence of other specific neurologic symptoms. In addition, non-specific symptoms, like pain without numbness, tingling and burning cannot be considered as diagnostic of CTS by themselves.

More severe cases of CTS may involve additional symptoms, including decreased sensation to pin or light touch in the first three digits or weakness or atrophy of the muscles of the thenar eminence, especially the abductor pollicis brevis. Thenar weakness or atrophy may reveal more acute or advanced nerve injury, in contrast with Hoffmann-Tinel's signor Phalen's sign, and in this case possibly creates the need for more aggressive treatment.

It is important to exhaust every possible effort to objectively verify the diagnosis of CTS before considering surgery. It has been showed that patients who have undergone carpal tunnel surgery with normal or near normal pre-surgical nerve conduction test results have worse outcomes than those with electro-diagnostic evidence of median nerve entrapment across the carpal tunnel, even though some evidence disagree. A rare method of therapeutic and diagnostic challenge test can be a steroid injection performed into the carpal canal. If the patient experiences improvement in symptoms for weeks or months after the injection but then relapses, surgery may be considered as a solution for carpal tunnel release. Further diagnostic evaluation can be performed by an appropriate specialist for the patients with a negative response [2]. 
Therapists should also investigate other clinical problems that may possibly relate to work exposure [6], such as tendonitis, in cases where CTS is not diagnosed by clinical criteria and nerve conduction testing. In addition, the patient should be referred to an appropriate specialist to eliminate the possibility of having other neurologic causes of tingling in the hands.

\subsection{Associated conditions}

Because of the functional and structural changes caused by CTS or other forms of polyneuropathy that lead to the syndrome, the median nerve becomes very sensitive to different compressive conditions. One of the associated conditions is hereditary neuropathy with hypersensitivity to pressure, which is a hereditary sensory motor form of neuropathy that is central and repetitive. The symptoms of the particular neuropathy, which include paralysis and paresthesias of the nerve trunk, usually appear after the age of 20. The symptoms, which usually occur after an injury or extended compression, often deteriorate and become more frequent. The condition is characterized by a disorder affecting the myelin of peripheral nerve fibers with stretching of the distal motor latency, which causes focal thickening of myelin in some zones of the nerve.

Another associated condition is the double constriction syndrome, which is based on the evidence that direct compression on the path of a nerve makes it more vulnerable than if the compression was located more peripherally. An appropriate clinical examination in addition with an electrophysiological study can determine whether the compression location is direct or peripheral and thus the treatment can be applied to the main compression location [5].

\section{Diagnosis}

Diagnosis CTS for patients reporting abnormal sensation like tingling or numbness of the hand and fingers, should include several steps [1], as described below. At first, the appropriate specialist should have the initial discussion with the patient regarding the symptoms and consider possible pathological conditions associated with the described symptoms. At this phase of the diagnosis it is important to conduct challenge tests, to be able to determine the etiology of the condition.

Some of the most common challenge tests are the following:

- Tinel sign - this test shows whether the patient feels tingling and numbness of the hands and fingers, when the specialist manually taps the palmar side of the wrist at the level of the median nerve. In that case the test is positive.

- Phalen sign - during this test the patient must perform maximum active flexion of the wrist for 1 minute, while having the elbow extended. If paresthesia appears in the area of the median nerve, the test is considered positive.

- Paley and McMurphy test - the particular test shows the presence or absence of paresthesia or pain triggered by the manual pressure about 1 centimeter from the median nerve.

- Compression test with wrist flexed - during this test the specialist uses his two fingers to apply pressure on the median region of the carpal tunnel, while the wrist is being flexed at 60 degrees, the elbow is extended and the forearm is supinated. In case of the appearance of paresthesia in the area of the median nerve, the test is positive.

Also, the presence of acroparesthesia at night, which includes tingling, numbness, swelling or pain, perceptible in the first three fingers, is considered one of the most sensitive tests.

The next step is to perform the Weber test, which evaluates the severity of the nerve compression, and analyze the strength of the thenar muscles innervated by the median nerve. The specialist then has to decide if additional examinations, such as nerve conduction velocity test (Error! Reference source not 
found.), are necessary. It is especially important to perform the nerve conduction velocity test, in cases where carpal tunnel decompression surgery is considered [7].

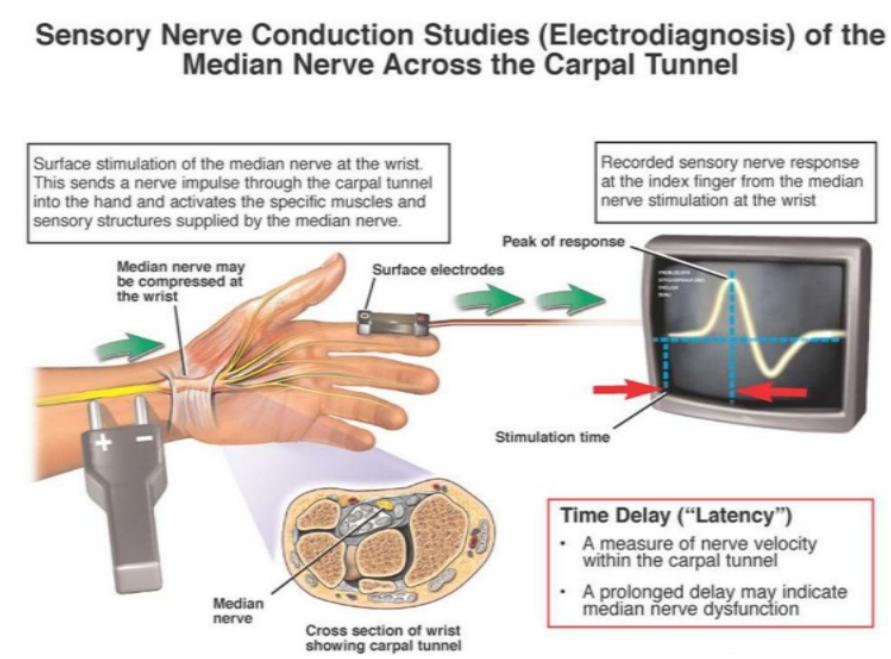

Figure 2: Nerve conduction study (adopted from [8])

The clinical diagnosis of CTS is validated by the nerve conduction study with high degree of reliability, when there is evidence of slowing of sensory and motor fibers of the median nerve across the carpal tunnel. In case all the symptoms of the patient indicate a positive clinical picture of CTS, while the nerve conduction test results are negative, the physician should consider other clinical diagnoses like tendonitis, pronator syndrome or cervical radiculopathy. The final step of the diagnosis procedure is to propose an appropriate treatment adjusted to the location, the cause and the severity of the condition.

\section{Treatment}

CTS patients can benefit from condition-specific guidelines that help make the treatment more effective and relieve the symptoms quickly. One of the most important guidelines is to avoid repetitive wrist and hand motions if possible and stop using vibratory tools that cause deterioration of the symptoms, such as jackhammers, floor sanders etc. Also, patients have to consider changing their work conditions, like changing the position of the wrists while working on the computer or start using wrist supports.

\subsubsection{Prevention}

Common musculoskeletal work-related injuries lead to CTS in improper workplace conditions. In order to prevent such injuries, the workspace and the equipment that used have to be at the right height and distance for the hands, so that the work is performed without straining the wrists. Computer users must maintain correct posture and ensure that keyboard position allows the wrist to lay comfortably without bending. Regular breaks also help lower the risk of swelling.

Other ways to prevent CTS is to perform tasks that use different muscle movements and try to do the hand and wrist motions without unnecessary tension. Exercise, like flexing and bending the wrists, after tasks demanding repetitive movements can reduce the negative effects of these tasks. Keeping the muscles warm makes them less likely to get hurt, so it is important to keep the hands warm at work, sometimes by wearing gloves. 


\subsubsection{Splints}

Splints are being used to support and brace the wrist, keeping it in a neutral position so that the nerves and tendons can recover. They are known to reduce repetitive flexion and rotation of the wrist and relieve mild swelling of the soft tissue or tenosynovitis. Splinting the wrist is usually more effective when it is performed within three months of the manifestation of the symptoms and when it is adjusted to the symptoms and the preferences of the patient. In addition, wearing the splints full time provides more effective improvement of the symptoms and electrophysiologic measurements.

\subsubsection{Surgery}

Surgery is the last resort for CTS patients and should only be considered in severe cases, when conservative measures do not alleviate the symptoms and other types of treatment do not have the expected result within at least six months of therapy [9]. The outpatient procedure for surgery includes a regional anesthesia that causes numbness to the wrist and hand area and a small incision in the palm that facilitates a division of the transverse carpal ligament and its overlying structures and enlarges the carpal tunnel. After the procedure the wrist needs to be splinted for three to four weeks. Possible surgery-related complications include injury to the palmar cutaneous or recurrent motor branch of the median nerve, tendon adhesion, hypertrophic scarring and laceration of the superficial palmar arch. Other possible complications are post-operative infection, hematoma, arterial injury, stiffness, and reflex sympathetic dystrophy. Recurrence of the symptoms has been observed, in cases that the carpal tunnel release was unsuccessful, which can be a result of incorrect or incomplete diagnosis.

\subsubsection{Medication}

Non-steroidal anti-inflammatory drugs (NSAIDs), such as aspirin, ibuprofen and other pain relievers, are used to relieve the symptoms of CTS and to control the pain. Cortisone injections or corticosteroids in a pill form may also help reduce swelling in more severe cases of the syndrome [5].

The injections of anesthetic combined with a corticosteroid into or near the carpal tunnel can be used for diagnosis and therapy. There is the risk of injuring the median nerve with the needle by performing a direct injection into the carpal tunnel. Other risks include causing intratendinous injection and tendon rupture or dysesthesias. As in surgery, wrist splinting is also recommended after an injection. If the first injection is successful, a second injection can be performed after a few months, although if another injection is needed, surgery should be considered as the next option.

\subsubsection{Ultrasound therapy}

Ultrasound therapy is possibly beneficial for the management of CTS, although more studies are needed to confirm that. Some studies have shown that twenty sessions of carpal tunnel ultrasound therapy administered over approximately seven weeks contribute in significantly greater reduction of symptoms at two weeks, seven weeks, and six months [10]. On the other hand, other studies doubt the benefit of this particular treatment for CTS.

\subsubsection{Physical Therapy}

A physical therapist can prescribe exercises to strengthen the wrist and the hand. Alternative approaches that can help relieve CTS symptoms include massage, yoga, ultrasound, chiropractic manipulation, and acupuncture.

\subsection{Traditional Physical Therapy}

Exercises for mobility and grip strength and pain reduction are the first two steps for a patient with reported loss of functionality due of CTS. The most common exercises recommended for the 
conservative management of the symptoms of CTS are tendon gliding of the finger flexor tendons and nerve gliding of the median nerve [11]. Studies that included measurements of the carpal tunnel pressure have shown that occasional exercise of active wrist and finger motion lasting about one minute lowers the pressure within the carpal tunnel [12]. Gliding exercises involve moving the fingers in a specified pattern to help the tendons and nerves glide more smoothly through the carpal tunnel. While there is some evidence that gliding exercises can help relieve symptoms when used alone, these exercises appear to work better in combination with other treatments such as splinting.
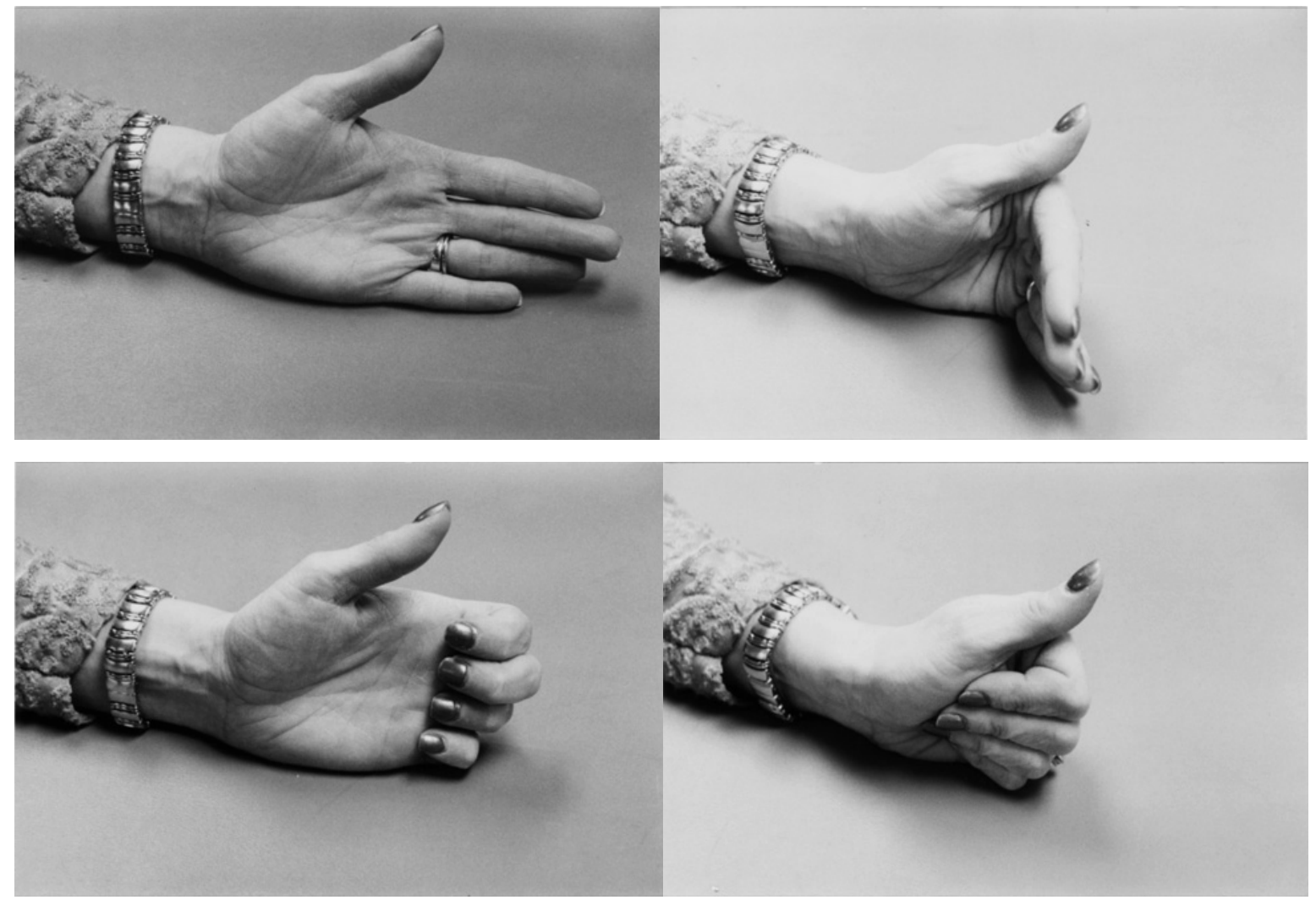

Figure 3: Common physiotherapy exercises for CTS treatment (adopted from [11])

Also manual therapy integrates a patented form of instrument-assisted soft tissue mobilization that gives the opportunity to the therapists to accurately detect and treat scar tissue and restrictions that effect normal function. Manual therapy techniques like active release technique and myofascial release are hands-on techniques that release tight tendons and musculature.

In the aggregate, physiotherapy is responsible for:

- Reducing pressure or inflammation, pain and loss of strength in your hand, wrist or arm

- Deciding whether a wrist splint is appropriate for the specific case

- Providing an individual program to improve functionality and increase the mobility of the wrist

- Instructing the patient to perform effective stretching exercises to prevent or minimize future symptoms appearance

- Providing specific advice on returning to work or normal everyday activities

\subsection{Computer-aided, game-based therapy}

A popular trend in today's game industry is to abandon conventional devices, such as keyboards, mice and joysticks in favor of mechanisms that detect the natural body motion, such as those employed by Nintendo's Wii and Microsoft's Kinect sensors. The purpose of these systems is to create the illusion to 
the user of being a part of the virtual environment by translating natural body movements to game movements according to the game scenario. Interactive technologies like video games with motionbased input devices create an interesting alternative way to perform prescribed exercises at home via rich graphical and multimodal game contexts used to motivate players. As a result of exercising within meaningful game contexts, the quality of home-based self-initiated therapy is expected to improve.

Although computer games are considered a form of entertainment for younger people, their primary purpose can extend to much more than entertainment. The process of memorizing the rules of the game, of developing the tactics and making quick decisions helps players improve reflexes, cognition, attention, self-control, and motor abilities [13]. A special category of games, called serious games, have already been used as training simulation for professionals in the field of the military, health care and engineering. Games involving challenges and rewarding systems can motivate users to learn or perform different tasks for the purpose of the game that wouldn't be normally do on their own.

Therefore, serious games have also found application in the field of health care and help resolve or at least mitigate frequent health problems, such as severe mobility impairments, especially those that occur due to neurological and musculoskeletal problems. An example of such condition that causes deficiencies leading to mobility impairment and limitations in everyday activities is the multiple sclerosis, a chronic progressive neurological disease that affects the communication capacity of nerve cells in the brain and the spinal cord. Another example of such condition, which often creates similar difficulties in the movement and also usually contributes in the loss of balance, is the stroke. It involves disorders of the blood supply to the brain and subsequently leads to a partial loss of brain functionalities, since the affected area of the brain stops functioning. The aftermath of such condition can be decreased reaction time, the loss of range of motion, disordered movement organization and impaired force generation, thus affecting the patient's ability for self-sufficient and independent life [14].

In addition, an important benefit of the game-based physical therapy is that the technology allows the patients that require regular physical therapy sessions to perform prescribed exercises at home, so that they can avoid frequent visits to treatment centers. Furthermore, patients don't usually feel attached to the rehabilitation exercise program and lose their interest easily, especially when the results are not prompt, since the exercises consist of repetitive and monotonous tasks. Accordingly, it is very important to configure appropriate environments for the performance of the physical therapy exercises that will be able to motivate the patients [15] and facilitate the completion of repetitive, tedious tasks efficiently, so that the patients can stay dedicated to the program and have better recovery prospects [16]. In the case of CTS, the gliding exercises, which are also repetitive movements of the wrist and fingers that are commonly prescribed, can be successfully implemented in an engaging game environment with interesting features keeping the patient amused while performing therapy.

The last decade there have been several approaches [17], [18], and [19] to take advantage of the emerging motion sensor technology in the fields of physiotherapy and rehabilitation. The variety of the sensors and the constant optimization of their potentiality and features make them convenient for the therapy of different motor disabilities. Many of the attempts are mostly academic research [20] [21] that includes some prototypes of video games using sensors for physiotherapy treatment, but there are also commercial products developed by technological companies.

VirtualRehab Hands [14], a mini-gaming platform developed by Virtualware, is using the Leap Motion sensor to aid recovery from health problems such as Parkinson's disease and stroke (Error! Reference source not found.). According to Virtualware, their platform is among the first rehabilitation suites to 
be classified as a medical device, under the EU's Medical Device Directives. The platform has been tested by patients and physiotherapists in installations in Europe, Latin America and the Middle East.

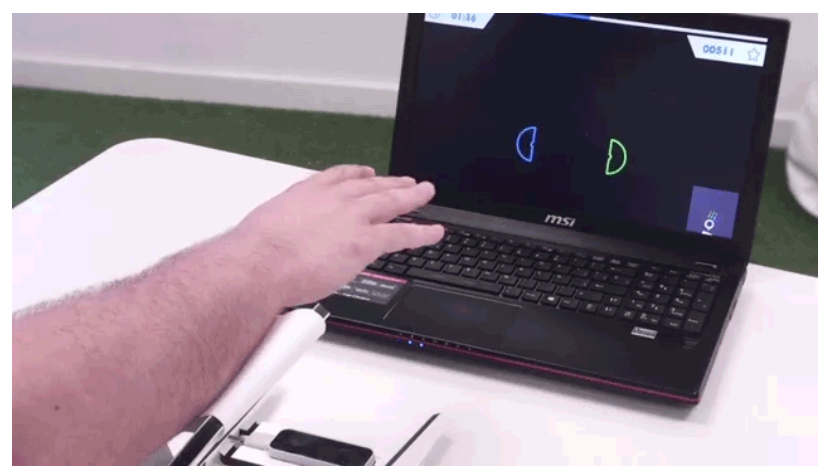

Figure 4: Virtual Rehab hand rehabilitation game (adopted from [14])

The games include exercises that help patients with motor disabilities and movement difficulties to preserve and regain upper and lower limb motor functionality. Additionally, the platform displays guidelines for the correct execution of the exercises and store the execution information or recording to provide feedback to the patient and allow the remote monitoring of the patients performance and progress by the therapist.

That same company has also developed the VirtualRehab-Body platform that employs the skeletal tracking and motion detection technology of Microsoft Kinect to detect and capture the movements of the patients within a game environment. The games include exercises that help patients with motor disabilities and movement difficulties to preserve and regain upper and lower limb motor functionality. Additionally, the platform displays guidelines for the correct execution of the exercises and store the execution information or recording to provide feedback to the patient and allow the remote monitoring of the patients performance and progress by the therapist.

The serious game "Kinect therapy - Boat Driving" developed by X-TECH Games team [22] utilizes the Kinect motion sensor to motivate the patients with different motor disabilities to perform their prescribed physical therapy exercises, but also urges casual users to train and gain body functionality improvement (Error! Reference source not found.). The game, involves the gathering of buoys in the sea by a boat driven by the user. The buoys score points and have to be gathered within certain time limits. These elements of time limit, score and rewarding instigate the users to execute the exercises often and for longer time. The user can operate the boat by performing rowing movements of the hands.

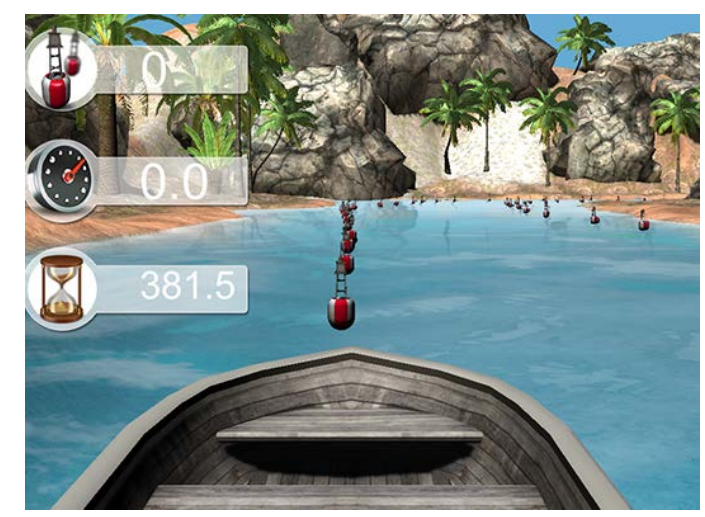

Figure 5: "Kinect therapy - Boat Driving" game by X-TECH Games team(adopted from [22]) 
Another commercial game platform that utilizes the Leap Motion controller for the purpose of rehabilitation and physical therapy is Visual Touch Therapy software program by the company Ten Ton Raygun [23]. The platform aims to make the therapy fun and engaging, while allowing the monitoring and assessment by the therapists to be easier. The target group of these therapeutic games is mostly patients that had stroke, but also individuals with spinal cord injuries, head injuries and nerve damage.

The Leap Motion Controller supports various movements and gestures, like swipes, taps, pushes and pulls, grabbing, grasping and side to side arm movements. Therefore, it gives the opportunity to the developers to implement a wide range of exercises. The games combine typical video game visual style, rewarding system with prompt feedback and various exercises for stroke rehabilitation, including hand and eye coordination challenges. The game scenario involves the Rocket Dog avatar that advances through the game levels and gains new abilities, such as speed, strength and rocket pack (Error! Reference source not found.).
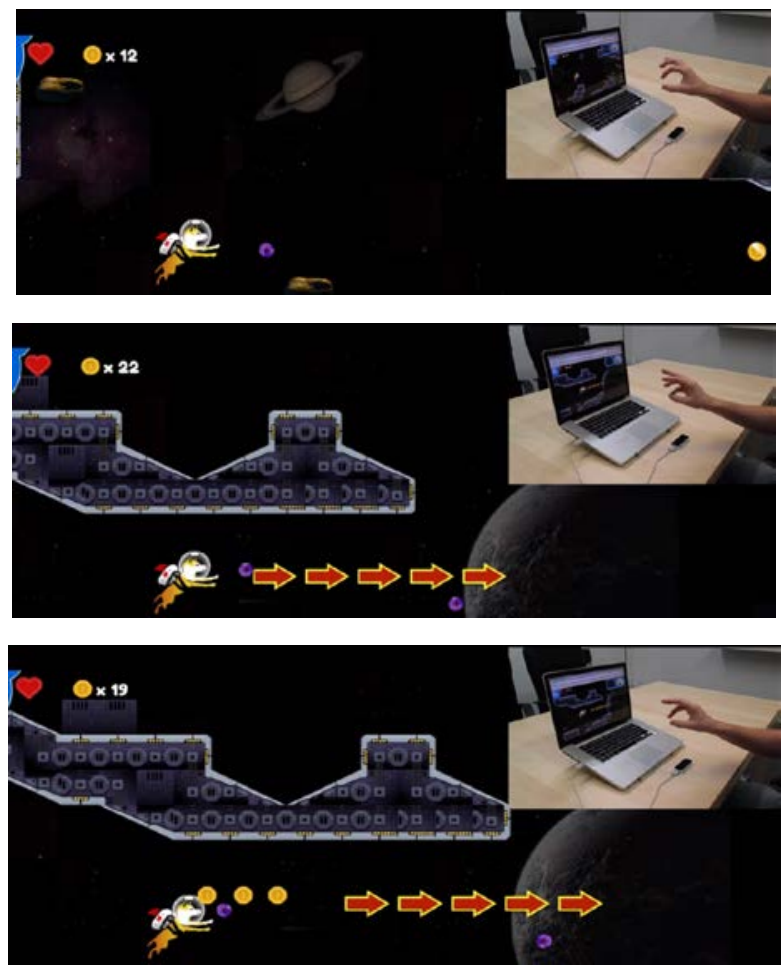

Figure 6: -“Rocket dog” stroke rehabilitation game that utilizes the Leap Motion sensor (adopted from [23])

As can be seen in the examples above, there are many attempts to develop physical therapy and rehabilitation platforms with the use of Kinect and Wii sensors. However, the Leap Motion sensor, despite the potential it offers in accuracy and portability, is still new in game development industry and especially in the field of therapeutic applications. The idea of developing a physical therapy game platform for the CTS with usage of Leap Motion controller seems very promising, due to increased accuracy of the sensor compared to other sensors, and quite original, since similar application examples haven't been found in the literature research.

\section{REFERENCES}

[1] "Physical Therapist's Guide to Carpal Tunnel Syndrome." [Online]. Available: http://www.moveforwardpt.com/symptomsconditionsdetail.aspx?cid=9f3cdf74-3f6f-40ca-b641d559302a08fc. [Accessed: 05-Mar-2016]. 
[2] M. Chammas, J. Boretto, L. M. Burmann, R. M. Ramos, F. C. dos Santos Neto, and J. B. Silva, “Carpal tunnel syndrome - Part I (anatomy, physiology, etiology and diagnosis)," Rev. Bras. Ortop. (English Ed., vol. 49, no. 5, pp. 429-436, 2014.

[3] "Carpal tunnel anatomy." [Online]. Available: https://www.mayoclinic.org/diseases-conditions/carpaltunnel-syndrome/multimedia/carpal-tunnel-anatomy/img-20007899.

[4] “Carpal tunnel syndrome - Treatment." [Online]. Available: http://www.nhs.uk/Conditions/Carpaltunnel-syndrome/Pages/Treatment.aspx. [Accessed: 01-Jan-2017].

[5] A. J. Viera, "Management of carpal tunnel syndrome," Am. Fam. Physician, vol. 68, no. 2, pp. 265-272, 2003.

[6] T. Gregory, D. Chamblin, J. Firestone, A. Friadman, H. Chirstopher, P. Douglas, M. Kliot, R. Lawrence, E. Thomas, B. Nicholas, D. Michael, T. Kjerulf, K. O’Bara, S. Carlson, and J. G, “Work-Related Carpal Tunnel Syndrome Diagnosis and Treatment Guideline," Washingtonworkingsolutions. Net. Washington State Department of Labor \& Industries, pp. 1-18, 2017.

[7] M. W. Keith, V. Masear, K. C. Chung, K. Maupin, M. Andary, P. C. Amadio, W. C. WattersIII, M. J. Goldberg, R. H. HaralsonIII, and C. M. Turkelson, "Clinical Practice Guideline on the Diagnosis of Carpal Tunnel Syndrome," 2007.

[8] R. Garaka, "Nerve conduction study," Health and Medicine, 2012. [Online]. Available: https://www.slideshare.net/garakarabel/nerve-conduction-study.

[9] D. Kostopoulos, "Treatment of carpal tunnel syndrome: A review of the non-surgical approaches with emphasis in neural mobilization," J. Bodyw. Mov. Ther., vol. 8, no. 1, pp. 2-8, 2004.

[10] A. H. Bakhtiary and A. Rashidy-Pour, "Ultrasound and laser therapy in the treatment of carpal tunnel syndrome," Aust. J. Physiother., vol. 50, no. 3, pp. 147-151, 2004.

[11] S. L. Michlovitz, "Conservative Interventions for Carpal Tunnel Syndrome," J. Orthop. Sport. Phys. Ther., vol. 34, no. 10, pp. 589-600, Oct. 2004.

[12] L. M. Rozmaryn, S. Dovelle, E. R. Rothman, K. Gorman, K. M. Olvey, and J. J. Bartko, "Nerve and tendon gliding exercises and the conservative management of carpal tunnel syndrome," J. Hand Ther., vol. 11, no. 3, pp. 171-179, Jul. 1998.

[13] A. M. D. C. Souza, M. a Gadelha, E. a G. Coutinho, and S. R. Santos, “A video-tracking based serious game for motor rehabilitation of post-stroke hand impairment," SBC J. 3D Interact. Syst., vol. 3, no. 2, pp. 3746, 2012.

[14] "Virtualrehab." [Online]. Available: http://www.virtualrehab.info/.

[15] K. Lohse, N. Shirzad, A. Verster, N. Hodges, and H. F. M. Van der Loos, "Video Games and Rehabilitation," J. Neurol. Phys. Ther., vol. 37, no. 4, pp. 166-175, Dec. 2013.

[16] F. Anderson, M. Annett, and W. F. Bischof, "Lean on Wii: Physical rehabilitation with virtual reality Wii peripherals," Stud. Health Technol. Inform., vol. 154, pp. 229-234, 2010.

[17] A. Y. Wang, "Games for Physical Therapy," SIGCHI Conf., pp. 4-8, 2012.

[18] M. E. Kho, A. Damluji, J. M. Zanni, and D. M. Needham, "Feasibility and observed safety of interactive video games for physical rehabilitation in the intensive care unit: a case series," J. Crit. Care, vol. 27 , no. 2, p. 219.e1-219.e6, Apr. 2012.

[19] A. de Mauro, "Virtual Reality Based Rehabilitation and Game Technology," in 1st International Workshop on Engineering Interactive Computing Systems for Medicine and Health Care (EICSMed 2011), co-located 
with the ACM SIGCHI Symposium on Engineering Interactive Computing Systems (EICS 2011), 2011, pp. 48-52.

[20] M. Khademi, H. Mousavi Hondori, A. McKenzie, L. Dodakian, C. V. Lopes, and S. C. Cramer, "Free-hand interaction with leap motion controller for stroke rehabilitation," in Proceedings of the extended abstracts of the 32nd annual ACM conference on Human factors in computing systems - CHI EA '14, 2014, pp. 16631668.

[21] L. Geurts, V. Vanden Abeele, J. Husson, F. Windey, M. Van Overveldt, J.-H. Annema, and S. Desmet, "Digital games for physical therapy: fulfilling the need for calibration and adaptation," in Proceedings of the fifth international conference on Tangible, embedded, and embodied interaction - TEI '11, 2011, vol. 53, p. 117.

[22] "Kinect Physical Therapy - Boat Driving." [Online]. Available: http://x-tech.am/kinect-physical-therapyboat-driving/.

[23] A. Gupta, "AXLR8R Spotlight: Making Physical Therapy Fun with Ten Ton Raygun," 2017. [Online]. Available: http://blog.leapmotion.com/axlr8r-spotlight-making-physical-therapy-fun-with-ten-tonraygun/. 